Instituto Internacional de Investigación y Desarrollo Tecnológico Educativo INDTEC, C.A.

DOI: https://doi.org/10.29394/scientific.issn.2542-2987.2016.1.1.11.190-211

OAI-PMH: http://www.indteca.com/ojs/index.php/Revista Scientific/oai

\title{
Competencias Docentes para la Construcción del Conocimiento de los Estudiantes de Educación Primaria
}

\author{
Autores: Wilmer José Pérez Rojas \\ Universidad Nacional Experimental "Rafael María Baralt", UNERMB \\ elprof 7@hotmail.com \\ Eglis Coromoto Quintero Gómez \\ Universidad Nacional Experimental "Rafael María Baralt", UNERMB \\ eglis2311@hotmail.com \\ Zulia, Venezuela
}

\section{Resumen}

La presente investigación tuvo como propósito ofrecer un referente teórico sobre las competencias docentes que optimice la construcción de conocimientos por los estudiantes de las escuelas primarias ubicadas en la parroquia Marcelino Briceño, municipio Baralt, para cumplir con ello, se sustentó teóricamente en los aportes de autores como Benavides (2008), Caldemón (2007), Díaz (2007), Alles (2006) entre otros. La metodología utilizada fue descriptiva, proyectiva con diseño de campo, transeccional, no experimental. La población censal estuvo conformada por 39 docentes pertenecientes a las instituciones mencionadas. La técnica fue la encuesta con un instrumento tipo cuestionario de escala múltiple. La validez se determinó mediante el juicio de expertos, la confiabilidad se obtuvo a través del cálculo del Coeficiente Alfa de Cronbach, alcanzando un valor de 0,83. Se concluyó que los docentes presentan debilidades en relación a las competencias requeridas para la construcción del conocimiento de sus estudiantes, pues continúan desempeñándose como simples transmisores de información. Se recomendó estar abierto a los cambios curriculares, actuar como mediadores de aprendizaje, desarrollar una práctica situada. Además se generó un referente teórico destinado a ofrecer a los docentes un conjunto de lineamientos para desarrollar competencias.

Palabras clave: competencias docentes; construcción del conocimiento; educación primaria. 


\title{
Teaching Skills for Construction of Knowledge of Primary Education Students
}

\begin{abstract}
This research was aimed to provide a theoretical reference on teaching skills to optimize the construction of knowledge by students of primary schools in the parish Marcelino Briceño, municipality Baralt, to meet it, it was based theoretically on the contributions of authors as Benavides (2008), Caldemon (2007), Diaz (2007), Alles (2006) among others. The methodology used was descriptive, projective with field design, transeccional not experimental. The census population consisted of 39 teachers from the institutions mentioned. The technique was the survey with a questionnaire type of multi-scale instrument. The validity was determined by expert judgment, reliability was obtained by calculating Cronbach Alfa Coefficient, reaching a value of 0.83 . It was concluded that teachers have weaknesses in relation to the competencies required for the construction of knowledge of their students, then continue to perform as mere transmitters of information. It was recommended to be open to curriculum changes, act as learning, developing a practical set. In addition, a theoretical reference intended to offer teachers a set of guidelines for developing competencies was generated.
\end{abstract}

Keywords: teaching skills; construction of knowledge; primary education. 


\section{Introducción}

Las sociedades modernas se caracterizan por estar sometidas al cambio permanente, la incertidumbre y el aumento vertiginoso de la cantidad de información disponible. En función de estas características, los ciudadanos han de contar con competencias para procesar información, resolver problemas, así como para el aprendizaje permanente.

Desde esta perspectiva, Tejeda (2011:32) acota que el concepto de competencia es muy diverso, según el ángulo desde el cual se mire o el énfasis otorgado a uno u otro elemento, pero el más generalizado y aceptado es el de "saber hacer en un contexto". Este requiere de conocimiento (teórico, práctico o una mezcla de ambos), además de afectividad, compromiso, cooperación, cumplimiento, todo ello expresado en el desempeño, también de tipo teórico, práctico o combinados.

Según Gutiérrez (2011:28) las competencias son capacidades complejas que poseen distintos grados de integración, manifestándose en una gran variedad de situaciones en los diversos ámbitos de la vida humana, personal y social. Es decir, son expresiones de los diferentes grados de desarrollo personal, así como participación activa en los procesos sociales.

Ahora bien, señala la autora antes mencionada, en el ámbito mundial se manifiesta una diversidad de interpretaciones y propuestas del término competencia, aunque es importante precisar que en sus diferentes variantes se trata de incorporar una concepción más amplia, profunda de formación, al generar objetivos, tareas dirigidas a demostrar desde la totalidad, los elementos intervinientes en el proceso

De esa manera, se establece una unidad armónica de los elementos ejecutores e inductores de la autorregulación de la personalidad del profesor, proporcionando una alternativa de vinculación entre el proceso de formación y el mundo profesional, la cual es multidimensional porque abarca las diversas 
dimensiones del acto de desempeño acorde con las exigencias de idoneidad del contexto socio-laboral.

En consecuencia, la competencia profesional es una cualidad humana que se configura como síntesis dialéctica en la integración funcional del saber (conocimientos diversos, habilidades, hábitos, destrezas, capacidades, valores, actitudes), los cuales son movilizados en un desempeño idóneo a partir de los recursos personológicos del profesor.

Al respecto, Mitrani y otros (2009: 93) afirman que la competencia "es una característica subyacente en una persona, casualmente relacionada con una actuación exitosa en un puesto de trabajo". Dentro de las competencias exigidas a los profesores universitarios ocupan un papel preponderante las competencias académicas, las cuales son producto de diferentes procesos de aprendizaje, aunque no necesariamente hayan ocurrido en contextos escolares, por cuanto se nutren también de la práctica y la experiencia, incorporando en la persona rasgos diversos: intelectuales, cognitivos, afectivos, motivacionales, conductuales, sociales.

La dinámica e interacción de los rasgos antes mencionados van creando formas cada vez más complejas. Desde esa perspectiva, Tobón (2006: 51) señala que las competencias académicas del docente representan un saber hacer o saber actuar en forma responsable, validada en un contexto profesional particular, combinando, movilizando recursos necesarios, conocimientos, habilidades, actitudes para lograr un resultado óptimo dentro de su práctica diaria.

Al analizar lo antes expuesto, se infiere que la profesión docente debe reunir dos condiciones primordiales: flexibilidad para adaptarse a las nuevas situaciones surgidas a diario, generadas por los cambios producidos, tanto en la didáctica como en la pedagogía y polivalencia para orientarse por el pluralismo en la acción educadora. 
Al respecto, Cano (2009: 37) reconoce que el antiguo paradigma sobre la enseñanza llevado a cabo prácticamente en todo el mundo, está desapareciendo para plegarse a una praxis basada en la construcción del conocimiento en los estudiantes quienes asumen el proceso fundamental de su aprendizaje, en colaboración permanente con los demás, siendo el profesor un facilitador en dicha construcción. Desde esa perspectiva, se reconoce que la actividad mental llevada adelante por el estudiante en interacción con el contenido no asegura por si sola una construcción óptima de significados.

En Venezuela, el sistema educativo bolivariano se orientan hacia la búsqueda de un individuo que logre intervenir en los procesos de integración social y transformación de sus acciones en pro del bienestar, tanto individual como colectivo, para ello diversos sectores exigen cambios más profundos los cuales garanticen interacciones favorables del individuo con su entorno, mencionándose, según Ollarves (2008: 9), el desarrollo de actividades limitantes del proceso de adquisición de conocimientos, por cuanto las mismas son el reflejo de un escenario poco dinámico donde el docente condiciona la participación del estudiante, además el estricto apego al programa de estudio, sin dar oportunidad al participante de aportar sus necesidades e intereses.

En este sentido, se le exige al docente el dominio de competencias académicas, entre las que se incluyen: conocimiento de un cuerpo teórico coherente, así como una didáctica que le garantice un encuentro real con la construcción de los saberes por parte de sus estudiantes, la transversalidad, y las referencias permanentes del contexto, asumiendo la enseñanza como un cuerpo dialéctico constitutivo de su práctica, el cual debe ser construido a partir de unas bases teóricas específicas.

Al respecto, Ollarves (2008: 11) acota que en algunas instituciones educativas del estado Zulia, se lleva a cabo una praxis caracterizada por clases magistrales, memorización, donde el estudiante sólo se concreta en aprender para los exámenes, pero no tiene la posibilidad de generar procesos 
de construcción de conocimientos. Considerando lo antes señalado, esta realidad pareciera presentarse en el Municipio Baralt, concretamente en las escuelas primarias, ubicadas en la parroquia Marcelino Briceño, en donde existen docentes, quienes presentan debilidades relacionadas con el dominio de las competencias académicas requeridas para desempeñarse adecuadamente en este nivel educativo, pues aun cuando son profesionales de la educación, se resisten a proseguir estudios de actualización

En observaciones reiteradas del investigador en las instituciones seleccionadas, se evidenció que los docentes no permiten la interpretación de los contenidos al momento de una exposición o entrega de trabajos, pues estos deben ir textualmente como el libro o guía de estudio, asimismo, al momento de evaluar, no valoran su participación para la asignación de calificaciones evidenciándose un probable desconocimiento de éstos en cuanto al buen uso de las herramientas pedagógicas. Cabría preguntarse si esta situación se mantendría en caso de que los docentes prosigan estudios de actualización o busquen orientación sobre los nuevos enfoques pedagógicos, los cuales les permitan adquirir conocimientos vigentes sobre cómo ejercer su praxis diaria. Por esta razón, este estudio pretende analizar la relación existente entre las competencias académicas del docente y la construcción de conocimientos por los estudiantes de las escuelas primarias ubicadas en la parroquia Marcelino Briceño, municipio Baralt.

\subsection{Formulación del problema}

¿Cuáles serían las competencias del docente para la construcción de conocimientos por los estudiantes de las escuelas primarias ubicadas en la parroquia Marcelino Briceño, municipio Baralt? 


\section{Teoría y Conceptos}

\subsection{Objetivo general}

Ofrecer un referente teórico sobre las competencias docentes que optimicen la construcción de conocimientos por los estudiantes de las escuelas primarias ubicadas en la parroquia Marcelino Briceño, municipio Baralt

\subsection{Objetivos específicos}

Identificar los tipos de competencias académicas que poseen los docentes de las escuelas primarias ubicadas en la parroquia Marcelino Briceño, municipio Baralt.

Describir los elementos que conforman la construcción de conocimientos por los estudiantes las escuelas primarias ubicadas en la parroquia Marcelino Briceño, municipio Baralt.

Generar un referente teórico sobre las competencias docentes que optimicen la construcción de conocimientos por los estudiantes de las escuelas primarias ubicadas en la parroquia Marcelino Briceño, municipio Baralt.

Cabe resaltar, que para sustentar esta investigación se hizo necesario realizar una revisión bibliográfica y documental, de la cual surgió un conjunto de estudios referidos a las variables antes mencionadas, seleccionándose los siguientes: El estudio de Ramírez (2009) denominado "Competencias docentes desde la perspectiva andragógica en facilitadores de educación superior" tuvo como objetivo analizar las competencias docentes desde la perspectiva andragógica en los facilitadores de las facultades de Derecho de las universidades Rafael Belloso Chacín y Popular del Cesar.

También se tomó el estudio de Portillo (2010) titulado "Competencias docentes en las instituciones educativas del nivel inicial" el cual tuvo como propósito analizar las competencias docentes en las instituciones educativas del nivel de educación inicial del Municipio Escolar Maracaibo 5. 
De igual forma, se ubica el estudio de Serrano (2012) denominada "Competencias docentes y formación en valores en instituciones de educación secundaria" para determinar las competencias necesarias en el docente para la formación en valores en las instituciones seleccionadas ubicadas en el municipio Chacao, Distrito Capital.

Las investigaciones presentadas se seleccionaron, por cuanto ofrecen elementos teóricos relacionados con la variable competencias docentes indicando la manera como deben formarse para alcanzar un adecuado desempeño dentro del aula de clase, los cuales podrán tomarse para fundamentar la dimensión y los indicadores de la misma.

\subsection{Competencias docentes}

El profesional de la educación para desempeñarse en el nivel de educación primaria requiere poseer un conjunto de competencias, las cuales dan forma al perfil que debe cumplir el docente, pues estas no solo se refieren a la función didáctica, sino, además, comprenden otros roles a cumplir para desempeñarse adecuadamente. Según Alles (2011) el término competencias define aquellas características de personalidad devenidas en comportamientos que generan un desempeño exitoso o superan el puesto de trabajo. En otras palabras, son la aptitud del individuo para ejercer una misma función productiva en diferentes contextos con base en los requerimientos de calidad emprendidos por el sector productivo, la cual se logra con la adquisición y desarrollo de conocimientos, habilidades, capacidades, expresadas en el saber, hacer, es decir, la capacidad real para lograr un objetivo o un resultado en un ambiente laboral dado.

En ese marco de pensamiento, Macías (2009) expresa que las competencias constituyen características permanentes en las personas, las cuales se ponen de manifiesto cuando se ejecuta una tarea o se realiza un 
trabajo, están relacionadas con la ejecución exitosa en una actividad, sea laboral o de otra índole.

\subsubsection{Competencias de docencia}

Las competencias de docencia del educador, según Andara (2009: 43), son aquellas acciones ejecutadas para la realización de las funciones y tareas vinculadas con la labor académica para la conducción del proceso enseñanza aprendizaje a través de aplicación de la didáctica, así como la utilización de las nuevas tecnologías incorporadas como recursos instruccionales. En ese sentido, las competencias de docencia le permiten al profesor desarrollar una buena gestión instruccional.

Desde esa perspectiva, Bedoya (2008: 103) señala que el saber pedagógico representa el objeto como producto del proceso de enseñanza 
aprendizaje, por ello, el profesor tiene el compromiso de transformar el conocimiento científico disciplinar en un conocimiento posible de enseñar, es decir, realizar una transposición didáctica, facilitándole al estudiante la apropiación de su aprendizaje. Dentro de las competencias de docencia, existe un elemento esencial: el desarrollo del proceso enseñanza aprendizaje, el cual en opinión de Urías (2009: 113), ha sido caracterizado históricamente de formas diferentes, las cuales van desde ser factor instruccional, con un marcado énfasis en el papel central del profesor como transmisor de conocimientos, hasta las concepciones actuales en donde se asume como un todo integrado poniendo en relieve el papel protagónico del estudiante. De las acotaciones anteriores, se considera una característica principal del proceso la integración cognitivo-afectiva, instructiva-educativa como requisitos para contribuir a la formación integral de la personalidad del estudiante.

\subsubsection{Competencia de investigación}

Dentro de las competencias exigidas al docente actual se ubican las de investigación, por cuanto representa un modelo didáctico alternativo, el cual transforma su práctica educativa, al permitirle transferir soluciones para resolver problemas cotidianos mediante la aplicación de los diversos modelos epistémicos existentes.

De acuerdo con Villarroel (2010: 32), se puede concebir la función de investigación como el hacer intelectual encaminado a la construcción del conocimiento en las diversas esferas de la actividad humana, mediante instrumentos racionales y materiales, entendidos a lo largo del tiempo, dentro del rigor, así como los cánones aceptados como científicos, cuyo fin último es el progreso del conocimiento.

Flores (2010: 28), acota que la competencia de investigación no parte de la certidumbre ni la seguridad del conocimiento de su disciplina, sino, se caracteriza por los siguientes aspectos: 
- El docente investigador enseña desde la duda, por cuanto su finalidad no es el dominio sino la búsqueda incansable.

- El investigador sabe que su primera tarea es interpretar el fondo histórico, sociocultural que lo acompaña, así como los rasgos y características estructurales de la existencia empírica, tanto propia como de los estudiantes, con el propósito de aprender a escucharlos.

En ese sentido, las competencias investigativas del docente de educación primaria, son definidas por González (2008: 71) como "un conjunto de aptitudes, capacidades y suficiencias que se poseen para emprender 0 generar acciones investigativas". Asimismo, Thomson y otros (2009: 89) las considera "habilidades del docente para hacer converger el sujeto, así como las expectativas de aprendizaje planteadas por la educación".

De lo anterior se infiere una noción de competencias para investigar o producir el conocimiento, asociadas a funciones cognitivas estructuradas en bloques o fases (entrada, proceso y salida de información) como formación de conceptos, así como proposiciones, estructuración de los conocimientos, razonamiento y capacidades expresivas o comunicativas.

En ese sentido, Maureira (2010) hace referencia a tres aspectos fundamentales:

\subsubsection{Competencias tecnológicas}

Según Perozo (2010: 47) las competencias tecnológicas se ubican dentro de las llamadas básicas, las cuales se consideran fundamentales para convivir y desenvolverse en cualquier ámbito laboral, caracterizándose por ser la base sobre la cual se conforman el resto de las competencias.

De acuerdo con lo antes señalado, pueden existir dos formas para conocer sobre competencias tecnológicas, midiéndolas y consultando la opinión de quienes las poseen en cuanto al uso de tecnologías informáticas. Al respecto, Cabello (2008: 99), comenta que cuando se trata de tecnologías 
informáticas la noción de uso se torna más compleja pues no solamente se consideran aspectos vinculados con contenidos sino se agregan las múltiples posibilidades generadas en la interactividad.

En un sentido amplio, puede decirse entonces que los usos de estas tecnologías implican una praxis operativa a partir de la cual los usuarios efectivamente reelaboran contenidos conforme a su experiencia cultural. Al respecto, Ruíz y otros (2011: 63) mencionan las competencias tecnológicas a adquirir por los docentes:

- Competencias básicas en el uso de las TIC: se refieren a los elementos necesarios para el manejo y divulgación del conocimiento.

- Competencias en el uso de las TIC para la navegación: comprenden los elementos necesarios para la comprensión y gestión de recursos mediante redes (Internet)

\subsection{Construcción del conocimiento}

La construcción del conocimiento se refiere a la adquisición de los aprendizajes, por parte de los estudiantes, quienes deben relacionar su estructura cognoscitiva con los contenidos nuevos, adquiridos durante el proceso de formación profesional, la cual le permite transferir los saberes alcanzados a situaciones cotidianas.

De acuerdo con Porlan (2011: 112) la construcción del conocimiento es fundamentalmente una construcción social, que requiere de procesos psicológicos del individuo, por lo tanto, se debe observar a este en intervención, con los más expertos de su cultura para así estudiar cómo se va apropiando de esas interacciones y las va interiorizando. Por lo tanto, resulta necesaria la actividad docente de enseñar procesos y contenidos relacionados con las diferentes actividades planteadas en el aula, así como en el entorno del estudiante, por lo cual, la enseñanza debe enfatizar situaciones relativamente específicas. 
En ese sentido, Valbuena (2008) asocia la construcción del conocimiento con el enfoque constructivista del aprendizaje, por cuanto este considera al estudiante como quien elabora su realidad, o al menos la interpreta basándose en sus percepciones.

\subsubsection{Elementos de la construcción del conocimiento}

El constructivismo se refiere a una concepción de la enseñanza y el aprendizaje, el cual sin prescribir una metodología específica, enfatiza la construcción activa de significados por el estudiante sobre los contenidos curriculares. Para Sanhueza (2010: 111), reconoce la capacidad del ser humano de construir nuevas ideas o conceptos pasados, es decir, experiencias previas, en sus interacciones por el entorno. Asimismo, Driver (2011: 97) afirma que el aprendizaje constructivista recalca el papel esencialmente activo de quien aprende el cual se basa en las siguientes características:

- Importancia de los conocimientos previos, las creencias y motivaciones de los estudiantes.

- El establecimiento de relaciones entre los conocimientos para la construcción de mapas conceptuales y la ordenación semántica de los contenidos de memoria (construcción de redes de significado).

Dicho de otra manera, el estudiante construye sus conocimientos de la relación que establece con sus saberes previos, así como sus experiencias personales. Por ello, Norman (2012: 122) define el constructivismo como "un conjunto de teorías psicológicas las cuales conciben los procesos cognitivos como construcciones eminentemente activas, resultado de la interacción, del sujeto con el ambiente, los demás y consigo mismo". 


\section{Metodología}

El tipo de investigación fue descriptiva, pues en el estudio de la problemática objeto de indagación, referida a las habilidades del directivo y la satisfacción laboral de los docentes adscritos a las escuelas primarias estadales ubicadas en la parroquia Marcelino Briceño, municipio Baralt, estado Zulia, se evidenciaron sistemáticamente las manifestaciones de las dimensiones $\mathrm{e}$ indicadores en forma independiente, para reconocer las magnitudes de aparición de aquellas en la formación del problema.

Al respecto, Landeau (2007: 57) señala que "los estudios descriptivos buscan medir conceptos o variables, así como evaluar diversos aspectos de un universo con la finalidad de identificar las características. De igual forma, Hurtado (2010:56) expresa que el propósito de un estudio descriptivo "es exponer el evento estudiado, haciendo una enumeración detallada de sus características de modo tal que en los resultados se pueden obtener dos niveles de análisis, dependiendo del fenómeno y del propósito del investigador. Asimismo, los objetivos del estudio determinan el tipo de investigación a realizar señalándola como proyectiva, por cuanto el objetivo general del estudio determina la investigación a realizar, siendo en este caso ofrecer un referente teórico sobre las competencias docentes que optimicen la construcción de conocimientos.

Según Hurtado (2010: 117) mediante la investigación proyectiva se proponen soluciones a una determinada situación a partir de un proceso previo de indagación. Implica explotar, describir, explicar y proponer alternativas de cambio, más no necesariamente ejecutar la propuesta. Asimismo, Balestrini (2009: 8) considera que este tipo de estudio se orienta a:

Proporcionar respuestas o soluciones a problemas planteados en una determinada realidad: organizacional, social, económica, educativa... En este sentido, la delimitación de la propuesta final, pasa inicialmente por la realización de un diagnóstico de la situación existente y la determinación de las 
necesidades del hecho estudiado, para formular el modelo operativo en función de las demandas de la realidad abordada.

Por lo antes expuesto, esta investigación se consideró proyectiva pues ofrece un referente teórico sobre las competencias docentes que optimicen la construcción de conocimientos por los estudiantes de las escuelas primarias ubicadas en la parroquia Marcelino Briceño, municipio Baralt.

\section{Resultados del Análisis de Contenido.}

Para analizar los datos, el procedimiento aplicado fue totalizar los puntajes alcanzados por cada alternativa por indicador, posteriormente se dividió entre el total de la población de docentes multiplicado por el total de ítems que miden el indicador para extraer el porcentaje correspondiente al mismo, seguidamente esos porcentajes se promediaron para obtener los valores de cada dimensión. Asimismo, se procedió a extraer el promedio de las frecuencias relativas para determinar los correspondientes a cada variable. Estos pasos se realizaron con la finalidad de hacer más comprensible el análisis para el lector.

Además, se expone la opinión del investigador basada en los referentes teóricos previamente analizados, los cuales permitieron la elaboración de las conclusiones y recomendaciones de la investigación, con el fin de lograr una información científica para ofrecer un referente teórico. De igual manera, los resultados arrojados infieren que los docentes encuestados presentan debilidades relacionadas con la forma como vienen desarrollando sus funciones docentes, mostrando escaso dominio de esta competencia, por cuanto se evidencian dificultades al momento de aplicar un proceso didáctico en el cual se encuentren presentes las nuevas tecnologías como recurso instruccional. En contraste, Andara (2009) señala que las competencias de docencia son aquellas acciones ejecutadas para la realización de las funciones 
y tareas vinculadas con la labor académica para la conducción del proceso enseñanza aprendizaje a través de aplicación de la didáctica, así como la utilización de las nuevas tecnologías incorporadas como recursos instruccionales. Estas competencias le permiten al docente desarrollar una buena gestión basada en los principios de libertad académica y excelencia de la enseñanza.

Al respecto, Alfaro (2009) señala que la función de docencia se constituye fundamentalmente a partir de la acción del profesor quien promueve el desarrollo de las potencialidades de sus estudiantes y la construcción de saberes, facilitando, orientando, guiando, motivando, apoyándolo para lograr su formación continua en un ambiente donde este actúe, piense con libertad, sea creativo, crítico, analítico, razone, participe, colabore en dicho desarrollo.

\section{Conclusiones}

Al culminar el proceso de análisis e interpretación de los resultados obtenidos por la aplicación del instrumento construido para medir las variables competencias docentes y construcción del conocimiento a través de sus dimensiones e indicadores se presentan las siguientes conclusiones que dan respuesta a los objetivos de la investigación.

Para el primer objetivo específico, se determinó que los docentes presentan debilidades relacionadas con la forma como vienen desarrollando sus funciones docentes, mostrando escaso dominio de esta competencia, por cuanto se evidencian dificultades al momento de aplicar un proceso didáctico en el cual se encuentren presentes las nuevas tecnologías.

Asimismo, continúan desempeñándose como simples transmisores de información, propiciando en sus educandos la asunción de un rol pasivo como receptor, alejado de los parámetros establecidos en el Currículo Bolivariano donde se explicita que el desarrollo de la praxis docente debe dirigirse hacia la construcción de nuevos conocimientos. 
En relación al segundo objetivo específico, se determinó que los docentes no consideran los elementos propios de la construcción del conocimiento, al dejar a un lado la aplicación de estrategias de aprendizaje que permita a sus estudiantes adquirir los saberes necesarios para el logro de las competencias propuestas en el Currículo Bolivariano. De igual forma, se evidenció que éstos se mantienen dentro de un esquema de enseñanza transmisivo, en el cual el ofrece información y el estudiante la recibe pasivamente, sin brindarle la oportunidad de contextualizar los contenidos recibidos.

También se determinó que presentan debilidades relacionadas con la ejecución de una evaluación acorde con los parámetros establecidos en el Currículo Bolivariano, evidenciando la realización de un proceso evaluativo netamente tradicional, desfasado, en donde sólo se aplican instrumentos para conocer la memorización de los contenidos, pero no para una real construcción del conocimiento.

En relación al tercer objetivo específico, los resultados obtenidos evidenciaron debilidades en relación al dominio de las competencias docentes requeridas para optimizar la construcción de conocimientos por sus estudiantes, ofreciendo insumos para generar el referente teórico antes mencionado, y así dar respuesta al último objetivo de la investigación, el cual se centra en el fortalecimiento de una serie de competencias que el docente debe desarrollar para optimizar su praxis cotidiana.

Desde esa perspectiva, la competencia no es un simple saber hacer, el cual se identifica con habilidades concretas: va mucho más allá al suponer un alto nivel intelectual, pues permiten afrontar, regular adecuadamente un conjunto de tareas y situaciones, haciendo uso de nociones, conocimientos, informaciones, procedimientos, métodos, técnicas u otras competencias más específicas. De esa manera, en esta investigación se entiende la noción de 
competencias docentes en un sentido amplio, flexible, creativo, desde una concepción más cercana a la perspectiva cognitiva.

\section{Referencias}

Alfaro, M. (2009). Planificación del aprendizaje y la enseñanza. Editorial FEDUPEL. Caracas

Alles, M. (2011). "Desarrollo del talento humano basado en Competencias". Editorial Granica. Buenos Aires.

Andara, P. (2009). "Competencias del profesor universitario". Editorial Ecoe. Bogotá.

Balestrini, M. (2009). "Cómo se elabora el proyecto de investigación". Editorial Consultores Asociados. Caracas.

Bedoya, J. (2008). "Pedagogía ¿Enseñar a pensar? Reflexiones filosóficas sobre el proceso de enseñar”. Editorial Ecoe. Bogotá.

Benavides, T. (2008). "La actitud del docente desde el enfoque por competencias". Editorial Ariel. México.

Cabello, R. (2008). "Aproximación al estudio de competencias tecnológicas". Editorial Paidós. Barcelona. España.

Caldemón, F. (2011). "Construcción del conocimiento". Editorial Popular. Madrid.

Cano, E. (2009). "Cómo mejorar las competencias de los docentes". Editorial Graó. Barcelona. España.

Carrera, C. y otros (2011). "Modelo pedagógico para el desarrollo de competencias en Educación Superior". Revista Costa Rica.

Díaz, V. (2007). “Construcción del saber pedagógico". Fondo Editorial FEDUPEL. Caracas.

Driver, L. (2011). “Aprendizaje constructivista”. Editorial Muralla. Madrid. 
Flores, F. (2010). "Las competencias que los profesores de Educación Básica movilizan el desempeño profesional”. Revista Iberoamericana de Educación.

González, L. (2009). “Modelos de investigación en ciencias sociales”. Editorial ICFES. Bogotá.

Gutiérrez, A. (2011). "La formación del profesorado en la sociedad de la información". Editorial Gedisa. Barcelona. España.

Hurtado, J. (2010). "El proyecto de investigación". Sypal. Caracas.

Landeau, R. (2007). "Elaboración de Trabajos de Investigación”. Editorial Alfa. Caracas.

Macías, R. (2009). "Competencias y excelencia empresarial". Ediciones Siglo XXI. Buenos Aires.

Maureira, S. (2010) "Importancia del liderazgo en el docente". Editorial Gedisa. Barcelona, España.

Mitrani, A. y otros (2009). "Competencias y competitividad". Ediciones Siglo XXI. México.

Norman, A. (2007). “Aprender a aprender”. Editorial Paidós. Barcelona, España.

Ollarves, I. (2008). "Gerencia Educativa y Desempeño Docente en Educación Básica". Trabajo de Grado. Universidad Pedagógica Experimental Libertador. Maracaibo.

Perozo, J. (2010). "Competencias de docentes universitarios en tecnologías de la información y la comunicación para el uso del edublog". Trabajo de grado. Maestría en Ciencias de la Comunicación. Universidad del Zulia. Maracaibo.

Porlan, R. (2011). "Constructivismo y enseñanza de las ciencias". Editorial Diana. Colección Investigación y Enseñanza. Sevilla, España. 
Portillo, O. (2010). "Competencias docentes en las instituciones educativas del nivel inicial". Trabajo de Grado. Maestría en Gerencia Educativa. Universidad Dr. Rafael Belloso Chacín. Maracaibo.

Ramírez (2009). "Competencias docentes desde la perspectiva andragógica en facilitadores de educación superior". Trabajo de Ascenso. Universidad Nacional Abierta. Maracaibo.

Ruíz, D., y otros (2007). "Formación docente: teoría y práctica”. Editorial Herder. Barcelona, España.

Sanhueza, G. (2010) "El constructivismo”. Editorial Kapesluz. Buenos Aires.

Tejeda, R. (2011). "Las competencias y su relación con el desempeño y la idoneidad profesional". Editorial Popular. Madrid.

Thomson y otros (2009) "Internet y educación. Nuevos horizontes para la investigación". Fundación Telefónica. Madrid.

Tobón, S. (2006). "Formación basada en competencias". Editorial Ecoe. Bogotá.

Urías, L. (2009). "Perfil del docente desde el enfoque por competencias". Trabajo de Grado. Maestría en Gerencia Escolar. Universidad de Santiago. Santiago de Chile.

Valbuena, V. (2008). "Desarrollo de actividades en el aula y el proceso de construcción de conocimiento en alumnos de Educación Básica". Revista Omnia, Vol. 14, Núm. 3. Universidad del Zulia. Maracaibo.

Villarroel, C. (2010) "El constructivismo y su papel en la innovación educativa". Revista Educación y Desarrollo, № 20. Universidad de Guadalajara. México. 


\section{Wilmer José Pérez Rojas}

e-mail: elprof 7@hotmail.com

Nació en Pueblo Nuevo Estado Zulia, el 30/04/1983.

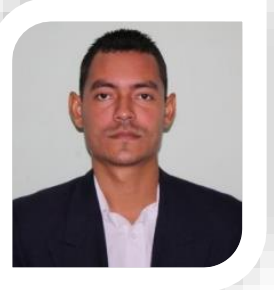

Estudiante de la Universidad Nacional Experimental "RAFAEL MARÍA BARALT" en el DOCTORADO EN EDUCACIÓN. Estudios de postgrado: Universidad "Valle de Momboy". Título obtenido: ESPECIALISTA

EN PLANIFICACIÓN EDUCACIONAL y en la Universidad Nororiental Gran "Mariscal de Ayacucho", título obtenido MAGISTER EN GERENCIA EDUCATIVA. Estudio de Pregrado: U.N.E.R.M.B. Titulo Obtenido: LICENCIADO EN EDUCACIÓN MENCIÓN INTEGRAL. Ha participado en cursos, talleres y simposios sobre "Fundamentos y principios en la evaluación de los aprendizajes" "Introducción a la Computadora". "Formación de Equipo de alto desempeño". "Ingles Instrumental" "Taller de Expresión Oral y Escrita." Participación de los Programas Fortalecimiento en la Familia." "Higiene y Seguridad Industrial" Proceso de Sensibilización Crecimiento y Liderazgo" Recapacitación y Actualización en el Nuevo Diseño curricular" Educación para los Valores Humanos." Transformación y Formación Educativa." Liderazgo Educativo" "Calidad Educativa" Sectorización e Investigación Social en Salud" "Proyecto Pedagógico de Aula" "Estrategias Didácticas para un aprendizaje Significativo" "Estrategias para la Enseñanza de la lengua oral y la Lengua Escrita". Maestro de Aula en la U.E.E. "Hilarión Alberto Viloria" San Rafael de El Tigre, Municipio Baralt Estado Zulia desde 2003 hasta la actualidad. "Profesor en la UBV desde 2009 hasta 2014, Profesor universitario en el IUTEHPI desde el 2015 hasta la actualidad. 


\section{Eglis Coromoto Quintero Gómez}

e-mail: eglis2311@hotmail.com

Nació en Pueblo Nuevo Estado Zulia, el 23/11/1986.

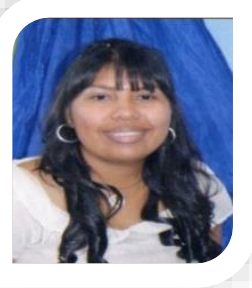

Estudiante de la Universidad Nacional Experimental "RAFAEL MARÍA BARALT" en el DOCTORADO EN EDUCACIÓN. Estudios de postgrado: UNIVERSIDAD NACIONAL EXPERIMENTAL RAFAEL MARÍA BARALT (UNERMB) título obtenido: MAGISTER SCIENTIARUM EN GERENCIA DE RECURSOS HUMANOS. Estudio de Pregrado: Universidad "ALONSO DE OJEDA" Título Obtenido: LICENCIADA EN EDUCACIÓN PREESCOLAR. Actualmente se desempeña como docente de Aula en el CEIB Don “JOSÉ MARIANO NÚÑEZ DE CÁCERES ubicado en San Rafael de El Tigre, Municipio Baralt Estado Zulia desde 2007 hasta la actualidad. 